\title{
Mathematical Modeling of the HIV-AIDS Epidemic
}

\author{
Roberto Arias, Kevin De Angeles, Shima Maleki, Reza R. Ahangar \\ Mathematics Department, Texas A \& M University Kingsville, Kingsville, USA \\ Email: roberto.arias@tamuk.edu, kevindeangeli@utk.gov, Maleki.4591@gmail.com,reza.ahangar@tamuk.edu
}

How to cite this paper: Arias, R., De Angeles, K., Maleki, S. and Ahangar, R.R. (2022) Mathematical Modeling of the HIV-AIDS Epidemic. Open Access Library Journal, 9: e7972.

https://doi.org/10.4236/oalib.1107972

Received: September 17, 2021

Accepted: February 25, 2022

Published: February 28, 2022

Copyright $\odot 2022$ by author(s) and Open Access Library Inc.

This work is licensed under the Creative Commons Attribution International License (CC BY 4.0).

http://creativecommons.org/licenses/by/4.0/

\begin{abstract}
A simple subset of an epidemic population model for HIV-AIDS can be split into the following: Susceptible, HIV-Infected, AIDS-infected, and Removed subsets (SHAR). We consider that the rate at which susceptible people become infected is proportional to the number of encounters between susceptible and infected individuals, which is proportional to the product of the two populations. A non-linear model is developed and its solution is produced with an Excel numerical approach using difference equations. Some solutions also are produced using MAPLE (CAS). Further research analysis, refining the model and qualitative analysis is the goal of this research.
\end{abstract}

\section{Subject Areas \\ Mathematical Analysis}

\section{Keywords}

Epidemics, Susceptible, HIV Infected, AIDS Infected, Removed and Cured Continuous Dynamical Systems, Discrete Dynamical Systems

\section{Introduction and History}

\section{HIV Cause:}

Human Immunodeficiency Virus is a virus that attacks the immune system of humans. It will develop into AIDS, or Acquired Immune Deficiency Syndrome. There is not cure for HIV-AIDS disease yet, but it is possible to control the virus with medication and avoid its spread.

Where the HIV-AIDS virus came from: HIV-AIDS virus has its origins in African monkeys and apes, HIV started as a disease affecting them. Later, the virus changed and was able to infect humans. A 2014 study suggests that the disease crossed over to humans more than a century ago, became endemic in 1920s Congo, and then traveled to Haiti in the 1960s and to the United States 
later. The disease became widespread in the West in the 1980s (see [1] [2] [3]).

White blood cells are our body's protection against any infection including HIV virus. This virus invades a certain kind of white blood cell, the CD4-positive T cell, and creates damages on the cell. The virus then makes copies of itself and spreads throughout the host body, infecting more $\mathrm{T}$ cells. Over time, healthy T cells go into decline while HIV-infected cells increase and weaken the immune system and evolve to AIDS.

A mathematical model for HIV infection had begun to be developed in the late 1980 using SIS and SIR models. These models have since been used to describe many other human virus infections, such as Hepatitis B and C, influenza, etc. [4]. Recently a mathematical model is proposed for the dynamics of HIV/AIDS with incorporation of weak $\mathrm{CD}^{+} \mathrm{T}$ cells in which assumed in several categories: uninfected $\mathrm{CD} 4^{+} \mathrm{T}$ cells, infected $\mathrm{CD} 4^{+} \mathrm{T}$ cells, and virus [5].

Before we attempt to model the HIV-AIDS Epidemic, it is natural for us to first gain some insight as to what we are going to be studying. Let us first look at where the disease came from. Scientists identified a type of chimpanzee in Central Africa as the source of HIV infection in humans. They believe that the chimpanzee version of the immunodeficiency virus (called simian immunodeficiency virus, or HIV) most likely was transmitted to humans and mutated into HIV when humans hunted these chimpanzees for meat and encountered their infected blood. Studies show that HIV may have jumped from apes to humans as far back as the late 1800s. HIV stands for Human Immuno-Deficiency Virus. Unlike some other viruses, the human body cannot get rid of HIV completely. So, once you have HIV, you have it for life. HIV attacks the body's immune system, specifically the CD4 cells (T cells), which help the immune system fight off infections. If left untreated, HIV reduces the number of CD4 cells (T cells) in the body, making the person more likely to get infections or infection-related cancers. Over time, HIV can destroy so many of T-cells that the body cannot fight off infections and disease. If HIV goes left untreated, it can lead to a more severe disease known as AIDS. AIDS stands for Acquired Immuno-Deficiency Syndrome. AIDS is the final stage of the HIV infection, and not everyone has HIV advances to this stage. AIDS is the stage of infection that the immune system, as the result of so much damage, causes the body to become vulnerable to opportunistic infections. When the number of your CD4 cells falls below 200 cells per cubic millimeter of blood ( 200 cells/cubic mm), the body has AIDS. People with AIDS need medical treatment to prevent death (see [6] [7]).

\section{The Statement of the Problem}

HIV and AIDS have been around for about 100 years, far longer than scientists have expected. People who do not have any of these two terminal diseases are very susceptible if they have contact with an HIV infected person. While it is not difficult to produce differential equations that are adequate to model this epidemic, as some exist already, it is always beneficial to add new perspectives and 
ideas on this epidemic. However, upon further exploration of this epidemic, we find that there exists no graphical representation of these two diseases. In this research, we will attempt to model the prevalence of contracting HIV or contracting AIDS over time. In this paper, we will show the representations of three separate experiments using Microsoft Excel and two separate experiments using Maple (CAS); after this research is represented, we will then show you one more experiment using Excel and three more using Maple, but in these particular experiments we will institute a new variable, a cure. The purpose of these representations is to try to predict the spread of the disease over a given period.

\section{Terms}

We are going to use specific letters to label our subsets of the population. In this paper, we shall use:

S: Susceptible, those people who are currently unaffected by either virus but may become infected.

H: HIV Infected, those people infected with the HIV virus.

A: AIDS Infected, those people infected with the AIDS virus.

$\mathrm{R}$ : Removed, those people isolated, cured, or permanently immune to the HIV and AIDS virus.

\section{Assumptions}

Before we start representing our data using different experiments, we must first institute postulates so our research and data has a particular framework to follow.

Assumption 1: We have a closed population, which means that no one enters, no one leaves, no one is born, and no one dies; so basically, the people that the population starts with are the exact same people that the population ends with.

Postulate 2: There is a positive integer $P$ such that:

$$
s(t)+h(t)+a(t)+r(t)=P \text {, for all } t
$$

Assumption 3: No one is permanently immune to the disease, and once a person has contracted HIV or AIDS, they can no longer be cured. The removal rate is so small that we can assume that $r(t)=0$ which gives us the following:

$$
s(t)+h(t)+a(t)=P
$$

Assumption 4: The rate of change of the susceptible population is negatively proportional to the rate of contact between the susceptible population with the HIV infected population, minus the rate of contact between the susceptible population, and AIDS infected population giving us the following equation:

$$
\frac{\mathrm{d} s}{\mathrm{~d} t}=-\frac{\mathrm{d} h(t)}{\mathrm{d} t}-\frac{\mathrm{d} a(t)}{\mathrm{d} t}=-\alpha h(t) \cdot s(t)-\beta a(t) \cdot s(t)
$$

where the constant $\beta$ is the AIDS infection rate and the constant $\alpha$ is the HIV infection rate. Notice that $s^{\prime}(t)$ is always negative (or possibly zero), since the number of people who have not yet caught the disease can only decrease with 
time $(t)$ (see [8] [9]).

\section{Computation of HIV and AIDS Epidemics}

Assumption 5: The rate of change of those infected with the HIV virus is proportional to the rate of contact between the HIV infected population and the AIDS infected population with those susceptible to the disease, which gives us the following equation:

$$
\frac{\mathrm{d} s}{\mathrm{~d} t}=s^{\prime}(t)=-\alpha h(t) \cdot s(t)-\beta a(t) \cdot s(t)
$$

and

$$
\frac{\mathrm{d} h}{\mathrm{~d} t}=h^{\prime}(t)=\delta_{1} \cdot h(t) \cdot s(t)+\delta_{2} \cdot a(t) \cdot s(t)=\left[\delta_{1} \cdot h(t)+\delta_{2} \cdot a(t)\right] \cdot s(t)
$$

where the constant $\delta$ is the constant infection rate of HIV for $\mathrm{d} h / \mathrm{d} t$. Notice that $h^{\prime}(t)$ always increases since the number of people who have the HIV virus will increase as those infected with the HIV virus and those infected with the AIDS virus interact with the susceptible population.

Now, as we scrutinize the previous axioms we can conclude that the rate of change of the AIDS population is negatively proportional to the rate of change of the susceptible population minus the rate of change of the HIV infected population, which gives us the following equation:

$$
\begin{aligned}
\frac{\mathrm{d} a}{\mathrm{~d} t} & =-\frac{\mathrm{d} s}{\mathrm{~d} t}-\frac{\mathrm{d} h}{\mathrm{~d} t} \\
& =-[-\beta a(t) \cdot s(t)-\alpha h(t) \cdot s(t)]-\delta_{1} h(t) \cdot s(t)-\delta_{2} a(t) \cdot s(t) \\
& =h(t) \cdot s(t)\left[\alpha-\delta_{1}\right]+a(t) \cdot s(t)\left[\beta-\delta_{2}\right]
\end{aligned}
$$

\section{Developing the System of Nonlinear Model}

If we assume that the epidemic started in a community with $P$ people with a positive number of HIV infected people and a positive number of AIDS infected people with $s_{0}=P-a_{0}-h_{0}$ susceptible, then we can produce the following system of differential equations by utilizing Equations (5.1), (5.2), and (5.3):

However, in order to produce a table and a graph that can approximate the prevalence and graph that can approximate the prevalence and outbreak of the epidemics over time we must first create a system of discrete difference equations from the continuous systems of differential Equation (6.1).

$$
\left\{\begin{array}{l}
\frac{\mathrm{d} s}{\mathrm{~d} t}=-\alpha \cdot h(t) s(t)-\beta \cdot a(t) s(t)=-\langle s(t), s(t)\rangle \cdot\langle\alpha \cdot h(t), \beta \cdot a(t)\rangle \\
\frac{\mathrm{d} h}{\mathrm{~d} t}=\delta_{1} \cdot h(t) s(t)+\delta_{2} \cdot a(t) s(t)=\langle s(t), s(t)\rangle \cdot\left\langle\delta_{1} \cdot h(t), \delta_{2} \cdot a(t)\right\rangle \\
\frac{\mathrm{d} a(t)}{\mathrm{d} t}=m \cdot s(t) h(t)+n \cdot s(t) a(t)=\langle s(t), s(t)\rangle \cdot\langle m \cdot h(t), n \cdot a(t)\rangle
\end{array}\right.
$$

where $m=\alpha-\delta_{1}$ and $n=\beta-\delta_{2}$. In a special case when two contagious factors delta are the same the system (6.1) will be 


$$
\left\{\begin{array}{l}
\frac{\mathrm{d} s}{\mathrm{~d} t}=-\alpha \cdot h(t) s(t)-\beta \cdot a(t) s(t) \\
\frac{\mathrm{d} h}{\mathrm{~d} t}=\delta[h(t)+a(t)] s(t) \\
\frac{\mathrm{d} a(t)}{\mathrm{d} t}=m \cdot s(t) h(t)+n \cdot s(t) a(t)
\end{array}\right.
$$

Let us assume that the initial conditions are given at time $t=0$,

$$
s(0)=s_{0}, h(0)=h_{0}, r(0)=r_{0} \text { and } s(0)=a_{0}
$$

There will be many different approaches to use this differential equations model. We will use a numerical computation approach to observe the behavior of the solution to this nonlinear system.

In addition to analytical analysis, we may use the computer algebra and programming methodology to simulate the model parameters.

\section{Discrete Form of HIV-AIDS Model}

To study the outbreak of the HIV-AIDS epidemic over time, we must first create a system of discrete difference equations from the continuous system of differential equations shown above.

At the partition points on the $\mathrm{t}$ axis for $j=1,2,3, \cdots$, the discrete difference equations are as follows:

$$
\left\{\begin{array}{l}
s[j]=-s[j-1]-\alpha h[j-1] \cdot s[j-1]-\beta a[j-1] \cdot s[j-1] \\
h[j]=h[j-1]+\delta(h[j-1]+a[j-1]) \cdot s[j-1] \\
a[j]=a[j-1]+s[j-1] \cdot h[j-1](\alpha-\delta)+a[j-1] s[j-1](\beta-\delta)
\end{array}\right.
$$

In the following experiment, we will use the spreadsheet computation method to show some numerical solution to the system. The system (7.1) can be solved by the method of discrete approximation, using the four initial conditions given in (6.3) and six other parameters involved in this model.

\subsection{Initial Conditions}

In our research, we conducted three separate experiments utilizing Microsoft Excel. Each experiment has its own parameters that are unique to that particular experiment. However, each experiment using Excel will have the same following initial conditions:

$N=25000$ people, $s(0)=s_{0}=24650$ people, $h(0)=h_{0}=250$ people, $a(0)=s_{0}=100$ people, $r(0)=r_{0}=0$ people, and $s(t)+h(t)+a(t)=P$ for all $t \geq 0$.

\subsection{Parameters for Excel Experiment (1)}

Assume that $\alpha=0.000012$, this means 12 out of every 100 thousand people with no disease may contract HIV. Let $\beta=0.00000003$. This means 3 out of every 100 million people with no disease contract AIDS. Also assume $\delta=0.000005$. This means the constant rate of infection for HIV is 5 out of every 1 million people. 


\subsection{Interpretation of the First Observation}

In this special experiment, we notice, with the parameters we used, that the susceptible population, $s(t)$, decreases over the 20-unit time-period. We can see from Table 1 that the susceptible population starts at 24,650 people and decreases

Table 1. This is a discrete computation for $S(t), H(t)$, and $A(t)$.

\begin{tabular}{|c|c|c|c|c|c|c|}
\hline$n$ & $S(n)$ & $H(n)$ & $A(n)$ & $S(n+1)$ & $H(n+1)$ & $A(n+1)$ \\
\hline 0 & 24,650 & 250 & 100 & 23,910 & 293 & 131 \\
\hline 1 & 23,910 & 293 & 131 & 23,069 & 344 & 164 \\
\hline 2 & 23,069 & 344 & 164 & 22,117 & 402 & 201 \\
\hline 3 & 22,117 & 402 & 201 & 21,049 & 469 & 241 \\
\hline 4 & 21,049 & 469 & 241 & 19,864 & 544 & 285 \\
\hline 5 & 19,864 & 544 & 285 & 18,567 & 626 & 333 \\
\hline 6 & 18,567 & 626 & 333 & 17,171 & 715 & 383 \\
\hline 7 & 17,171 & 715 & 383 & 15,697 & 810 & 437 \\
\hline 8 & 15,697 & 810 & 437 & 14,172 & 907 & 492 \\
\hline 9 & 14,172 & 907 & 492 & 12,628 & 1007 & 547 \\
\hline 10 & 12,628 & 1007 & 547 & 11,103 & 1105 & 602 \\
\hline 11 & 11,103 & 1105 & 602 & 9631 & 1199 & 654 \\
\hline 12 & 9631 & 1199 & 654 & 8244 & 1289 & 704 \\
\hline 13 & 8244 & 1289 & 704 & 6969 & 1371 & 749 \\
\hline 14 & 6969 & 1371 & 749 & 5823 & 1445 & 790 \\
\hline 15 & 5823 & 1445 & 790 & 4813 & 1510 & 826 \\
\hline 16 & 4813 & 1510 & 826 & 3941 & 1566 & 857 \\
\hline 17 & 3941 & 1566 & 857 & 3200 & 1614 & 884 \\
\hline 18 & 3200 & 1614 & 884 & 2580 & 1654 & 906 \\
\hline 19 & 2580 & 1654 & 906 & 2068 & 1687 & 924 \\
\hline 20 & 2068 & 1687 & 924 & 1650 & 1714 & 939 \\
\hline 21 & 1650 & 1714 & 939 & 1310 & 1736 & 951 \\
\hline 22 & 1310 & 1736 & 951 & 1037 & 1753 & 961 \\
\hline 23 & 1037 & 1753 & 961 & 819 & 1767 & 969 \\
\hline 24 & 819 & 1767 & 969 & 645 & 1778 & 975 \\
\hline 25 & 645 & 1778 & 975 & 508 & 1787 & 980 \\
\hline 26 & 508 & 1787 & 980 & 399 & 1794 & 984 \\
\hline 27 & 399 & 1794 & 984 & 313 & 1800 & 987 \\
\hline 28 & 313 & 1800 & 987 & 245 & 1804 & 989 \\
\hline 29 & 245 & 1804 & 989 & 192 & 1808 & 991 \\
\hline 30 & 192 & 1808 & 991 & 150 & 1810 & 992 \\
\hline 31 & 150 & 1810 & 992 & 118 & 1813 & 994 \\
\hline 32 & 118 & 1813 & 994 & 92 & 1814 & 995 \\
\hline 33 & 92 & 1814 & 995 & 72 & 1815 & 995 \\
\hline 34 & 72 & 1815 & 995 & 56 & 1816 & 996 \\
\hline 35 & 56 & 1816 & 996 & 44 & 1817 & 996 \\
\hline 36 & 44 & 1817 & 996 & 34 & 1818 & 997 \\
\hline 37 & 34 & 1818 & 997 & 27 & 1818 & 997 \\
\hline 38 & 27 & 1818 & 997 & 21 & 1819 & 997 \\
\hline 39 & 21 & 1819 & 997 & 16 & 1819 & 997 \\
\hline 40 & 16 & 1819 & 997 & 13 & 1819 & 997 \\
\hline
\end{tabular}

Table 1: Selected parameters-alpha $=0.000012$, beta $=0.00000003$, delta $=0.000005, \mathrm{~N}=$ 25,000. Figure 1: $\mathrm{S}(0)=24,650, \mathrm{H}(0)=250, \mathrm{~A}(0)=100, \mathrm{R}(0)=0$. 
to about 16,074 people over the 20 -year span. If we look at the HIV infected population, $h(t)$, in Figure 1 we notice that the population increases to slightly above 5000 people. Looking back at Table 1 we notice that the HIV Infected population increases from 250 people to about 5759 people over the 20 -year span. If we take a moment to look at the AIDS Infected population, $a(t)$, in Figure 1, we will notice that the AIDS-Infected population increased but seems to increase at about half the rate as the HIV Infected population. In Table 1, we see that the AIDS population increases from 100 people to about 3168 people. From the given parameters that we used in this first experiment on Microsoft Excel, we can see that this special epidemic spread over the 20-unit time period, but it did not completely exterminate the population. Since we assumed that there would be no cure in this special experiment, we can conclude that, if we extended the time-period, the susceptible population would eventually converge to 0 as the HIV and AIDS infected population converge to their maximums (see [9] [10]).

\subsection{Parameters for Excel Experiment (2)}

Assume that $\alpha=0.00002$. This means that 2 out of every 100 thousand people

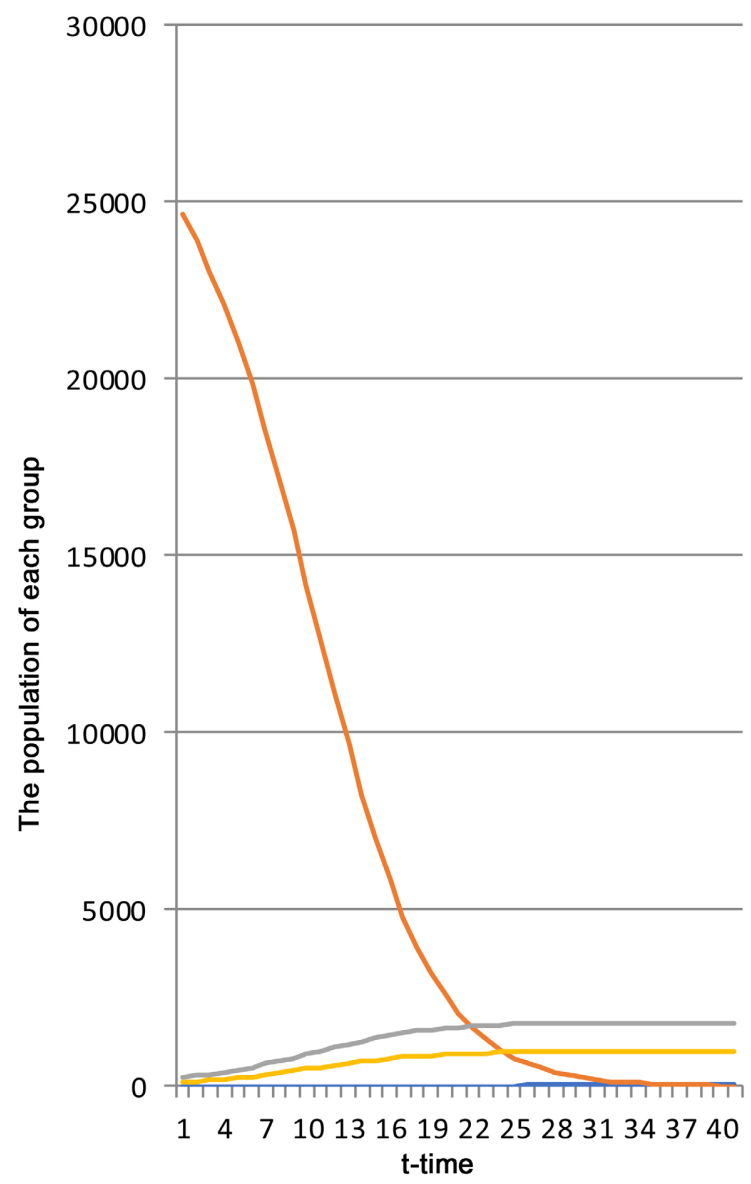

Figure 1. Graph of $S(t)$-Susceptible blue, $H(t)$-HIV Red, and $A(t)$-Aids Green. 
with no disease contract HIV. Let $\beta=0.00000005$. This means 5 out of every 100 million people with no disease contract AIDS. Also assume $\delta=0.0000085$. This means the constant rate of infection for HIV is 85 out of every 1 million people.

\subsection{Observation of the Computation for the Experiment (2)}

In this particular experiment, we notice, with the parameters we used, that the susceptible population, $s(t)$, decreases very dramatically over the 20-time period. We can see from Table 2 that the susceptible population decreases radically over

Table 2. Susceptible. HIV-Aids population.

\begin{tabular}{|c|c|c|c|c|c|c|c|c|}
\hline$n$ & $S(n)$ & $H(n)$ & $A(n)$ & $S(n+1)$ & $H(n+1)$ & $A(n+1)$ & & \\
\hline 0 & 24,650 & 250 & 100 & 24,527 & 332 & 150 & alfa & 0.00002 \\
\hline 1 & 24,527 & 332 & 150 & 24,364 & 444 & 213 & beta & 0.00000005 \\
\hline 2 & 24,364 & 444 & 213 & 24,147 & 597 & 293 & delta & 0.0000085 \\
\hline 3 & 24,147 & 597 & 293 & 23,858 & 801 & 399 & $\mathrm{n}$ & 0.00025 \\
\hline 4 & 23,858 & 801 & 399 & 23,476 & 1073 & 538 & $\mathrm{~m} 1$ & 0.00004 \\
\hline 5 & 23,476 & 1073 & 538 & 22,971 & 1433 & 721 & $\mathrm{~m} 2$ & 0.00002 \\
\hline 6 & 22,971 & 1433 & 721 & 22,312 & 1903 & 960 & & \\
\hline 7 & 22,312 & 1903 & 960 & 21,462 & 2511 & 1267 & & \\
\hline 8 & 21,462 & 2511 & 1267 & 20,383 & 3281 & 1657 & & \\
\hline 9 & 20,383 & 3281 & 1657 & 19,043 & 4238 & 2141 & & \\
\hline 10 & 19,043 & 4238 & 2141 & 17,427 & 5393 & 2724 & & \\
\hline 11 & 17,427 & 5393 & 2724 & 15,545 & 6738 & 3404 & & \\
\hline 12 & 15,545 & 6738 & 3404 & 13,448 & 8237 & 4162 & & \\
\hline 13 & 13,448 & 8237 & 4162 & 11,229 & 9822 & 4962 & & \\
\hline 14 & 11,229 & 9822 & 4962 & 9021 & 11,400 & 5760 & & \\
\hline 15 & 9021 & 11,400 & 5760 & 6961 & 12,872 & 6504 & & \\
\hline 16 & 6961 & 12,872 & 6504 & 5167 & 14,154 & 7151 & & \\
\hline 17 & 5167 & 14,154 & 7151 & 3702 & 15,201 & 7680 & & \\
\hline 18 & 3702 & 15,201 & 7680 & 2575 & 16,006 & 8087 & & \\
\hline 19 & 2575 & 16,006 & 8087 & 1750 & 16,596 & 8385 & & \\
\hline 20 & 1750 & 16,596 & 8385 & 1168 & 17,012 & 8595 & & \\
\hline 21 & 1168 & 17,012 & 8595 & 770 & 17,296 & 8739 & & \\
\hline 22 & 770 & 17,296 & 8739 & 504 & 17,487 & 8835 & & \\
\hline 23 & 504 & 17,487 & 8835 & 327 & 17,613 & 8899 & & \\
\hline 24 & 327 & 17,613 & 8899 & 212 & 17,695 & 8941 & & \\
\hline 25 & 212 & 17,695 & 8941 & 137 & 17,749 & 8968 & & \\
\hline 26 & 137 & 17,749 & 8968 & 88 & 17,784 & 8985 & & \\
\hline 27 & 88 & 17,784 & 8985 & 57 & 17,806 & 8997 & & \\
\hline
\end{tabular}

Table 2: This is a model for HIV-AIDS with no vaccination or removed subset of the population: $r(t)=0$. 
the given time period and drops well below 5000 people. If we look at Figure 2, we can see that the susceptible population starts at 24,650 people and decreases to about 2977 people over the 20 -year span. If we take a look over at the HIV infected population, $h(t)$, in Figure 2 we notice that the population increases substantially, near 15,000 over the 20-year span. Looking back at Figure 2 we notice that the HIV Infected population increases from 250 people to about 14,348 people over the 20-year span. If we take a look at the AIDS Infected population, $a(t)$, in Figure 2, we notice that the AIDS Infected population also increased a great deal, just as the HIV Infected population did, but we can still notice that the HIV population increased a lot more than the AIDS population. If we look at Figure 2, we see that the AIDS population increases from 100 people to about 7675 people. From the given parameters we used in this second experiment on Microsoft Excel, we can see that this particular epidemic spread very quickly over 20 years, and it seems to put a big dent in the susceptible population, dropping it to under 5000 people. Since, as before, we assumed that there would be no cure in this particular experiment, we can conclude that, if we extended the time period, the susceptible population would probably decrease to zero within the next few years, and the HIV and AIDS population would reach their maximums rather quickly.

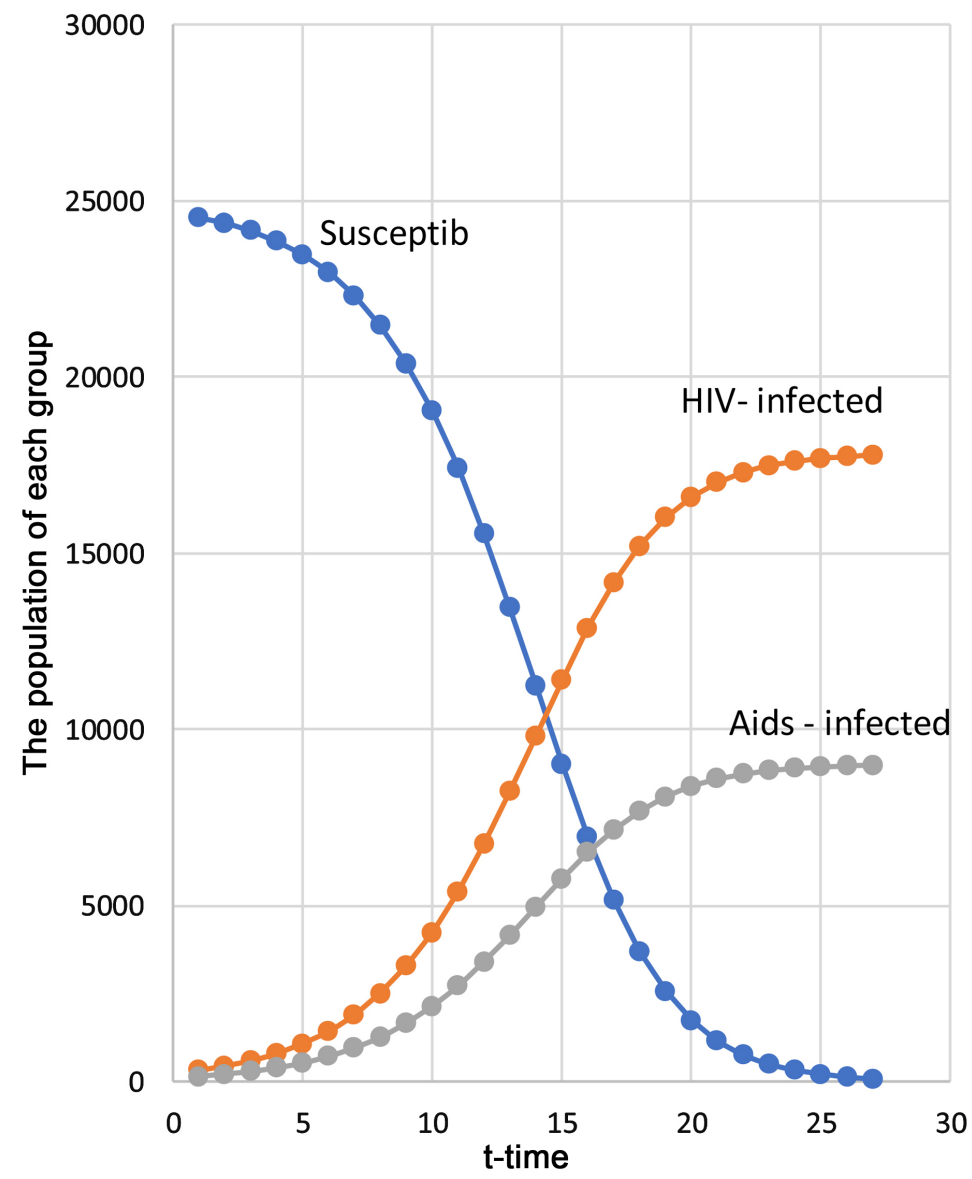

Figure 2. Portrait of susceptible, HIV, and Aids infection. 


\subsection{Analytical Attempt to Find the Exact Solution}

In the following nonlinear model, we consider distinct parameters coefficients and demonstrate that all other known models, then we will study some of the special cases of this general form.

$$
\left\{\begin{array}{l}
\frac{\mathrm{d} s}{\mathrm{~d} t}=-\alpha \cdot h(t) s(t)-\beta \cdot a(t) s(t) \\
\frac{\mathrm{d} h}{\mathrm{~d} t}=\delta_{1} \cdot h(t) s(t)+\delta_{2} \cdot a(t) s(t) \\
\frac{\mathrm{d} a(t)}{\mathrm{d} t}=m \cdot s(t) h(t)+n \cdot s(t) a(t)
\end{array}\right.
$$

Note that $m=\alpha-\delta_{1}$ and $n=\beta-\delta_{2}$. The system (6.1) and (6.3) are equivalent.

Two subsets of the populations HIV infected, and AIDS infected in general have different rates of transmitting the disease. That is all parameters in (6.1) to (6.3) are not equal and the nonlinear systems can be solved by numerical approximation and demonstrate the general; behavior of the system using the technology of computer algebra system (CAS) or spreadsheet.

However, for simplicity and checking the validity of the model, we may assume that individuals in the susceptible group become infected at equal rates by mixing with the subsets of the big population. That is, consider

$$
\alpha=\beta, \delta_{1}=\delta_{2} \text { and } m=n
$$

We can add another condition: $h(t)+a(t)=I(t)$, to (6.4) then, the system (7.3) will change,

$$
\left\{\begin{array}{l}
\frac{\mathrm{d} s}{\mathrm{~d} t}=-\beta[h(t)+a(t)] s(t) \\
\frac{\mathrm{d} h}{\mathrm{~d} t}=\delta[h(t)+a(t)] s(t) \\
\frac{\mathrm{d} a(t)}{\mathrm{d} t}=m \cdot[h(t)+a(t)] s(t)
\end{array}\right.
$$

Or, equivalently

$$
\left\{\begin{array}{l}
\frac{\mathrm{d} s}{\mathrm{~d} t}=-\beta \cdot I(t) \cdot s(t) \\
\frac{\mathrm{d} h}{\mathrm{~d} t}=\delta \cdot I(t) \cdot s(t) \\
\frac{\mathrm{d} a(t)}{\mathrm{d} t}=m \cdot I(t) \cdot s(t)
\end{array}\right.
$$

Adding the second and the third equation in (7.6) we will get

$$
\frac{\mathrm{d} I(t)}{\mathrm{d} t}=h^{\prime}(t)+a^{\prime}(t)=(\delta+m) I(t) s(t)
$$

With no removal function, using $s(t)=N-I(t)$ and $k=\delta+m$, we can produce the following SIR differential equations that can be solved by separation of variables. 


$$
\frac{\mathrm{d} I(t)}{\mathrm{d} t}=M \cdot I(t) s(t) \text { or }\left\{\begin{array}{l}
\frac{\mathrm{d} I(t)}{I(N-I)}=k \mathrm{~d} t \\
I(t)=I(0)
\end{array}\right.
$$

The well-known solution of Equation (6.7) is

$$
I(t)=\frac{N \cdot I(0)}{I(0)+(N-I(0)) \cdot \mathrm{e}^{-N k t}}=\frac{N}{1+\left(\frac{N}{I_{0}} N-1\right) \cdot \mathrm{e}^{-N k t}}
$$

This result shows that the total HIV plus AIDS infection over the time interval $[0, t]$ can be calculated.

\section{Maple Computation for Epidemic Experiment (1)}

Now we want to represent the same data using different parameters and a different software. For this, we utilized Maple Software (CAS). Maple is a computer algebra system, is a symbolic and numeric computing environment as well as a multi-paradigm programming language.

The nonlinear model (6.2) is constructed based on the assumption that the subpopulation of AIDS infected individuals is coming from the interactions between susceptible and HIV, not directly from HIV. To refine this model, we can assume:

Assumption 6: During the time-period of this study, we are assuming a certain constant proportion $\mathrm{p}$ of the HIV infected individual will develop AIDS.

$$
\left\{\begin{array}{l}
\frac{\mathrm{d} s}{\mathrm{~d} t}=-\alpha \cdot h(t) s(t)-\beta \cdot a(t) s(t) \\
\frac{\mathrm{d} h}{\mathrm{~d} t}=\delta[h(t)+a(t)] s(t) \\
\frac{\mathrm{d} a(t)}{\mathrm{d} t}=(\alpha-\delta) \cdot s(t) h(t)+(\beta-\delta) \cdot s(t) a(t)+p \cdot h(t)
\end{array}\right.
$$

Notice that in the Maple code (Figure 3), we replaced all Greek letters in this system by $\mathrm{k}, \mathrm{l}, \mathrm{m}$, and $\mathrm{n}$ for convenience.

\subsection{Results (Maple Experiment 1)}

Maple Software has its own spreadsheet, but it is more convenient to create a table like the one produced on Microsoft Excel and Maple can produce much more interesting graphical results. If we look above at the coding for the software,

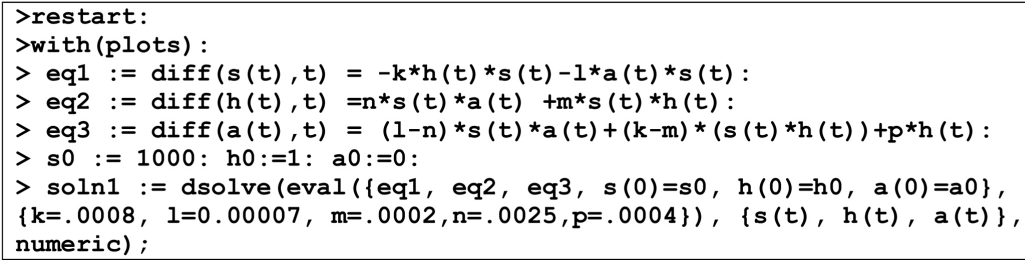

Figure 3. This is the updated code for Maple, you can see the three same equations, along with slightly modified initial conditions and new parameters for the rates of change. 
we notice that the parameters are not Greek letters, but instead, are English letters. The reason for this is that it is easier to input English letters into the software rather than inputting Greek letters to represent the rates of change. However, this does not mean that the graphs are wrong. If we take a moment to look at Figure 4(a), we can see that the susceptible population dramatically decreases to 0 extremely quickly. Within 20 years, the susceptible population diminishes to zero. If we look at Figure 4(b), we can see that the HIV Infected population, which only started with one person, converged to its peak very quickly and then plateaued once it reached its maximum value. Just as with the susceptible population, the HIV Infected population peaked in about a 20 -year span of time. If we move over to Figure 4(c) we notice that the AIDS Infected population also increased very rapidly in the first 20 years. However, unlike the susceptible and HIV population, the AIDS population does not seem to plateau after the first 20 years of time. If we look closely at the graphs, we can see that after the first two decades of time, the AIDS population still increased by about 20 people over the next 80 years of time. This could be a result of some people form the HIV infected population not taking care of themselves, which allows their number of T-cells to drop drastically causing them to succumb to the AIDS virus (see [10] $[11])$.

\subsection{Maple (Experiment 2)}

We knew that showing this epidemic only one time with the Maple software was not going to be adequate, so we restarted the Maple code and repeated the experiment using slightly modified initial conditions that you will notice in the updated code below. Also, just as we did with the Microsoft Excel experiments, we utilized new parameters for the rates of change within each separate equation.

The nonlinear model (6.2) it was constructed based on the assumption that the subpopulation of AIDS infected individuals is quarantined, and there is no

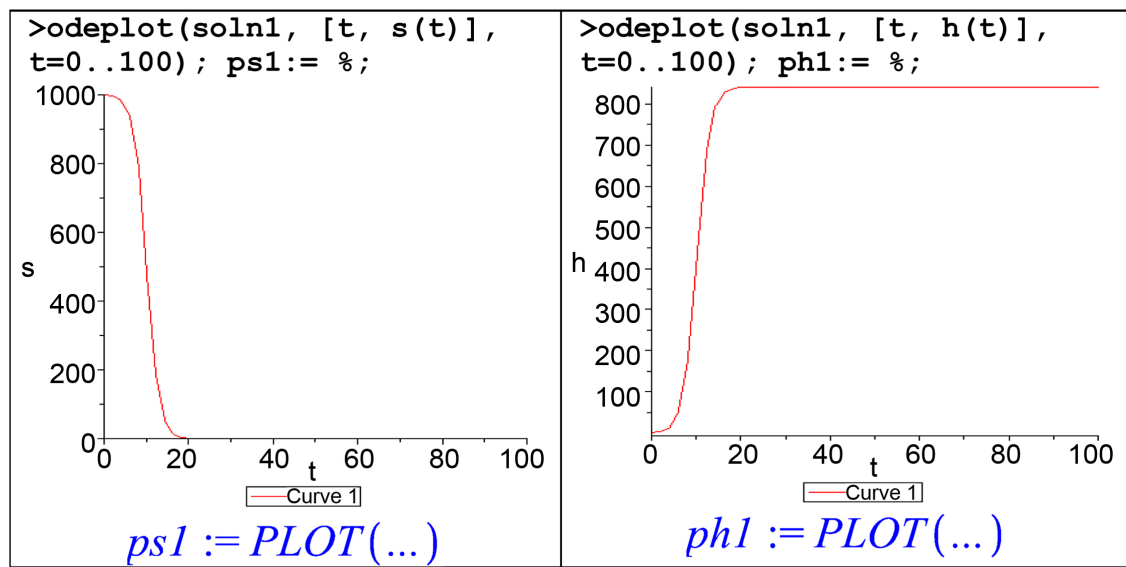

(a) (b)

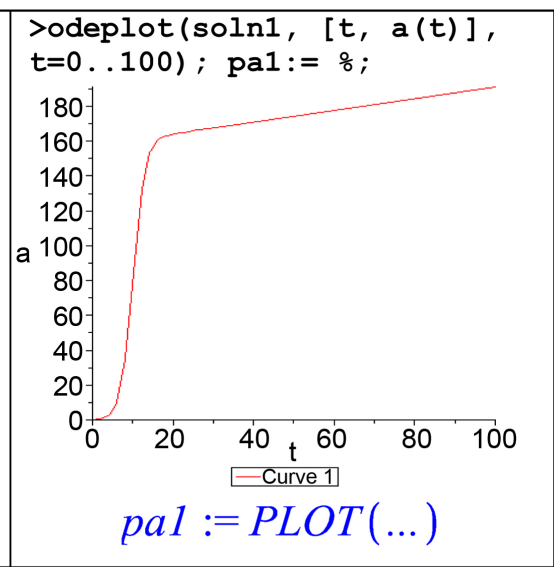

(c)

Figure 4. This is the code for Maple, you can see the three equations, along with initial conditions and parameters for the rates of change. (a) With this rate of fast virus transmission the susceptible population $s(t)$ tends to zero. (b) The HIV epidemic converging to the highest level. (c) This graph shows that the AIDS population increasing fast. 
interaction between susceptible and AIDs infected individuals.

To refine this model, we can assume:

Assumption 6: During the time-period of this study, we are assuming that a certain constant proportion $\mathrm{p}$ of the HIV infected individuals will develop AIDS.

Let us run the MAPLE code with different initial conditions and different values of parameters (Figure 5).

\subsection{Results for Maple Experiment (2)}

The new parameters and initial conditions produced the graphs shown above. If we take a moment to look at Figure 6(a), we can see the susceptible population dramatically decrease to 0 over a long period of time. Within 200 years, the susceptible population completely vanishes. Just as in experiment 1, the susceptible population goes to 0 , but this time it took 200 years, not 20 . If we look at Figure 6(b), we can see that the HIV Infected population, which only started with 1 person, shows little to no change at first, then it rapidly increases dramatically after about 75 years of dormancy. If we look closer at the susceptible population

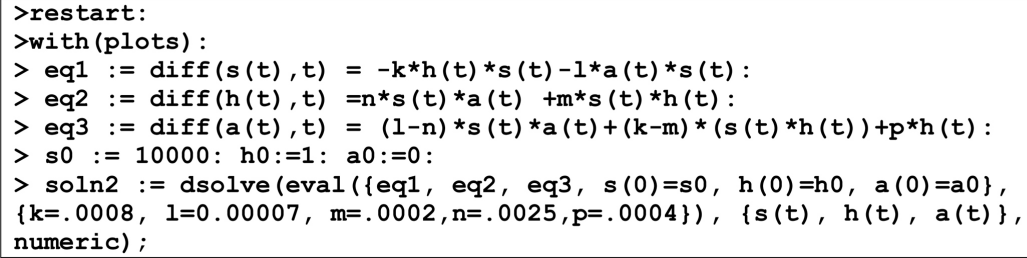

Figure 5. This is the updated code for Maple, you can see the same three equations, along with slightly modified initial conditions and new parameters for the rates of change.

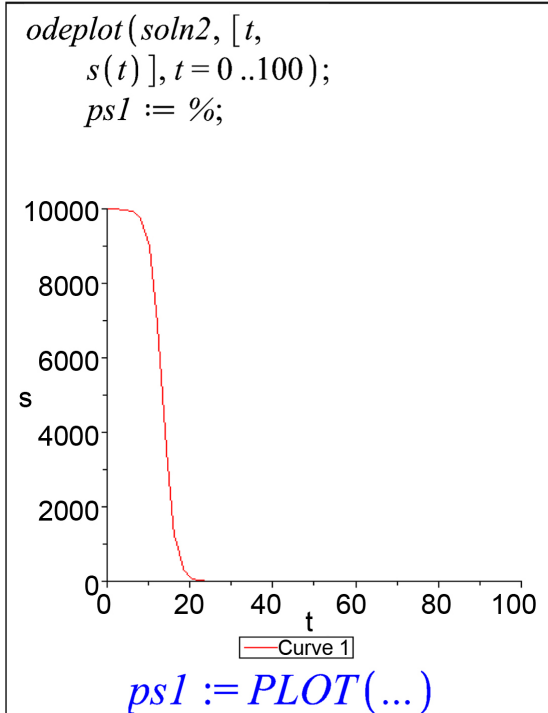

(a)

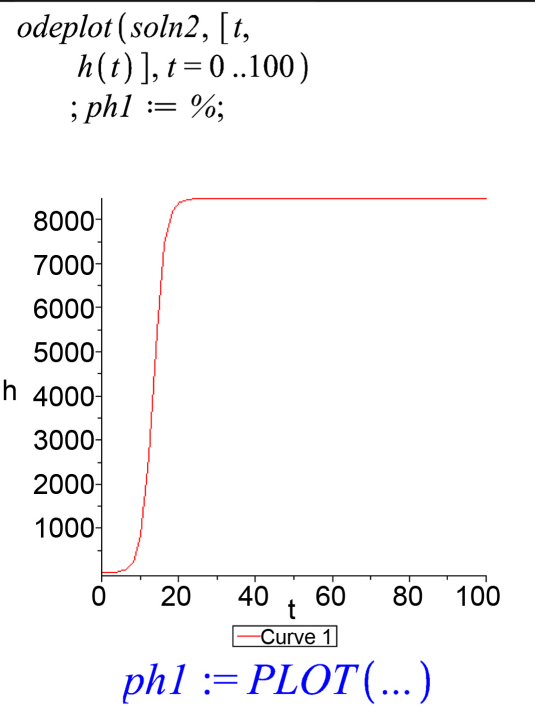

(b)

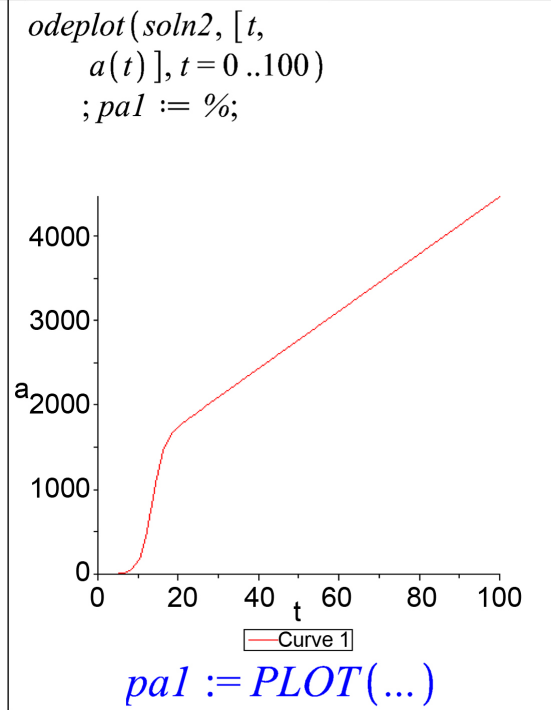

(c)

Figure 6. This is the updated code for Maple, you can see the three same equations, along with slightly modified initial conditions and new parameters for the rates of change. (a) With the new parameters the susceptible population still decreases rapidly over time. (b) The HIV population show little change at first, then rapidly increases after 75 years. (c) The AIDS population increases rapidly and converges at its peak. 
and the HIV population, we notice that their graphs almost look like reflections of each other. If we move over to Figure 6(c) we notice that the AIDS Infected population also increased very rapidly but only after about 150 years. As we can see upon further inspection of Figure 6(c), we notice that the AIDS population increases very rapidly and converges to its maximum very quickly. It is safe to say that our parameters are far from perfect, and it is quite difficult to produce rates of change that go hand in hand with those produced by Mother Nature. Upon scrutinizing and analyzing our data, we visualize where our experiment can be modified and greatly improved. Further improvement of the maple code is the goal.

\section{Conclusions}

It is very important to present a mathematical description of a natural phenomenon like HIV/AIDS epidemic.

We postulated all related assumptions and defined all parameters involved. The mathematical model presented in this investigation is a nonlinear ODE (ordinary differential equation). To solve a nonlinear system of ODE, one can choose either a numerical, graphical, or analytical approach.

The basic concept of mathematical modeling in epidemiology is the variety of subsets of the population and their fundamental interactions. A simple subset of the epidemic population model for HIV-AIDS is: Susceptible, HIV-Infected, AIDS-infected, and Removed subsets that we used $S(t), H(t), A(t)$, and $R(t)$.

Our numerical and graphical approaches demonstrate that with some certain conditions or choices of parameters, one HIV infected individual can transmit the virus to the entire susceptible population and can infect the entire population.

We did not consider the vaccination factor for modeling this epidemic model in the first few experiments. A reasonable function for removing the immune individuals who are not susceptible to the virus will be denoted by $r(t)$. The nonlinear system will be a system of four ODE equations (see reference [12]).

Once we added the removed population, we can see that it can play a role in determining the outcome of each unique, separate population that we focused on.

As we sit down and analyze our experiments and our results, we can conclude that an epidemic such as this one can play out in a few different ways. This why we reproduced several different scenarios including one where there is a cure and one where there is not. We know that some of our parameters are not exact, but the point of this research was to show several different possibilities. The end goal of our research is to improve the Maple code and produce more accurate results.

\section{Conflicts of Interest}

The authors declare no conflicts of interest. 


\section{References}

[1] Mayo Clinic (2015) HIV/AIDS. Mayo Foundation for Medical Education and Research.

[2] https://www.aids.gov/hiv-aids-basics/hiv-aids-101/what-is-hiv-aids/index.html

[3] Kaplan, J.E. (2021) WebMD: HIV-AIDS Overview: Reviewed by Jonathan E. Kaplan. https://www.webmd.com/hiv-aids/ss/slideshow-hiv-aids-overview

[4] Hill, A.L. (2018) Mathematical Models of HIV Latency. Current Topics in Microbiology and Immunology, 417, 131-156.

https://www.ncbi.nlm.nih.gov/pmc/articles/PMC6117215/

[5] Dutta, A. and Gupta, P.K. (2018) A Mathematical Model for Transmission Dynamics of HIV/AIDS with Effect of Weak CD4 ${ }^{+} \mathrm{T}$ Cells. Chinese Journal of Physics, 56, 1045-1056. https://www.sciencedirect.com/science/article/pii/S0577907317313436

[6] Curran, J.W. (1988) Epidemiology of HIV Infection and Aids in the United States. Science, 239, 610-611.

[7] Hoffman, M.S. (ed.) (1992) The World Almanac 1993. World Almanac Publishing Co., New York.

[8] Olenik, M. (1992) An Introduction to Mathematical Models into the Social and Life Science. Addison-Wesley Publishing Company, Boston.

[9] Adithyan, G.S., Rakshase, B. and Ekström, A.M. (2017) A Study on HIV Knowledge and Preventive Behavioral Practices among Female Sex Workers (FSW) in Mumbai, India. The Journal of Aids and HIV Research. https://academicjournals.org/journal/JAHR/edition/January 2017

[10] AIDS.gov. (2017) A Timeline of HIV/AIDS. AIDS.gov. https://www.hiv.gov/hiv-basics/overview/history/hiv-and-aids-timeline

[11] https://www.webmd.com/hiv-aids/ss/slideshow-hiv-aids-overview

[12] Ahangar, R.R. (2022) Computation, Modeling, and Simulation of HIV-AIDS Epidemics with Vaccination. Mathematics Department, Texas A \& M University, Kingsville. (To Be Appeared) 